\title{
Effets comparés des pratiques paysannes et des bonnes pratiques agricoles de gestion de la fertilité des sols sur les propriétés des sols et les rendements des cultures dans la zone sud soudanienne du Burkina Faso
}

\author{
Souleymane NACRO ${ }^{1}$, Souleymane OUEDRAOGO ${ }^{1}$, Karim TRAORE $^{1 *}$, \\ Estanislasse SANKARA ${ }^{1}$, Célestin KABORE ${ }^{2}$ et Bernard OUATTARA ${ }^{2}$ \\ ${ }^{1}$ Institut de l'Environnement et de Recherches Agricoles (INERA), CRREA Ouest Station de Farakoba 01 BP \\ 910 Bobo 01, Burkina Faso. \\ ${ }^{2}$ Direction régionale de l'A griculture de l'Hydraulique et des Ressources Halieutiques des Hauts Bassins, \\ Bobo-Dioulasso, Burkina Faso. \\ *Corresponding author, E-mail: karim_traore24@yahoo.fr, karim_traore@hotmail.com \\ Tel: +226703845 52; Fax: +22620970159
}

\section{RESUME}

Au Burkina Faso, $86 \%$ de la population active travaille dans l'agriculture. Cependant la production agricole a du mal à couvrir les besoins d'une population sans cesse croissante. Les raisons sont d'ordres climatiques et anthropiques. La diffusion et l'adoption de techniques efficientes de gestion de la fertilité des sols constituent un défi majeur pour une intensification durable des productions agropastorales. C'est dans le but de diffuser des pratiques de gestion durable des ressources naturelles dénommées bonnes pratiques agricoles (BPA) que des expérimentations ont été conduites de façon tripartite entre l'union des producteurs de coton du Burkina (UNPCB), l'Institut de l'Environnement et de Recherches Agricoles (INERA) et La FAO dans un champ école de producteurs de 2006 à 2008. Les traitements ont comparé les pratiques paysannes (PP) et les bonnes pratiques agricoles (BPA). Des données pédologiques et agronomiques ont été collectées au cours du programme afin de caractériser les facteurs ayant conduits au succès des bonnes pratiques agricoles. Les résultats montrent une nette amélioration du taux de matière organique, d'azote et surtout du phosphore assimilable des sols dans les parcelles sous bonnes pratiques agricoles. Les BPA ont engendré une augmentation significative des rendements grains du maïs et du coton. Les bonnes pratiques agricoles et les jachères annuelles affectent peu le $\mathrm{pH}$ du sol ainsi que le taux de $\mathrm{K}$ total. L'utilisation d'un amendement alcalin et d'une source de potassium seront nécessaires pour renforcer l'effet des bonnes pratiques agricoles.

(C) 2010 International Formulae Group. All rights reserved.

Mots clés: Sols, pratiques, fertilité, rotations, légumineuses, école.

\section{INTRODUCTION}

Le secteur agricole occupe environ $86 \%$ de la population active au Burkina Faso et représente plus du tiers du produit intérieur brut. Cependant, cette agriculture a du mal à couvrir les besoins alimentaires d'une population en pleine expansion. Seuls 30\% des ménages Burkinabé parviennent à satisfaire leurs besoins alimentaires et nutritionnels (Traore et Stroosnijder, 2005). Cette situation s'explique d'une part par des conditions climatiques aléatoires 
(pluviométrie et température) et d'autre part, par des systèmes d'exploitation agricole peu adaptés conduisant à une dégradation continue de la capacité productive des terres (Vlaar, 1992).

La zone sud soudanienne du Burkina Faso possède les sols les plus fertiles du pays mais ceux-ci sont en dégradation continue. Les systèmes de production agricole de cette partie du pays sont relativement intensifs avec le coton comme culture motrice. Ces systèmes allient à la fois la production cotonnière, la production céréalière et l'élevage à la faveur de la culture attelée (systèmes mixtes cotoncéréales-élevage). Une des conséquences de l'accroissement des moyens de travail (équipements agricoles) et de la population agricole, est la saturation des terroirs villageois, offrant de moins en moins de possibilité pour la pratique de la jachère, pratique de rétablissement de la fertilité de la terre la plus connue des producteurs (INERA, 1994, 2003). Le maintien de la fertilité des terres agricoles devient de plus en plus difficile, alors que l'exportation des éléments fertilisants (principalement par les cultures) et la minéralisation de la matière organique du sol s'accentuent (Sedogo, 1981, 1993 ; Zougmoré et al., 1999; Thiombiano, 2000). Le recours à des amendements organiques dans ce contexte est limité à cause de la faiblesse des effectifs de bovins intégrés dans les exploitations d'une part, et d'autre part, par le temps de séjour réduit des animaux dans les exploitations agricoles.

Le recours à des pratiques conservatrices des ressources naturelles constitue de nos jours la seule alternative pour la durabilité des systèmes de production. Plusieurs techniques de gestion de la fertilité des sols ont été mises au point par la recherche et testées avec succès en milieu réel (Sedogo, 1993 ; Lompo, 1995 ; Zombré et al., 1999 ; Traoré et Stroosnijder, 2005, Traoré et al., 2007). Cependant, ces techniques restent méconnues de nombreux potentiels utilisateurs. Les techniques de gestion durable de l'espace agricole ont été regroupées sous forme d'un paquet technologique unique dénommé les bonnes pratiques agricoles (BPA). La diffusion et l'adoption de ce paquet constituent un défi majeur dans un contexte d'intensification des productions agropastorales. Dans le cadre de la collaboration entre l'Institut de l'Environnement et de Recherches Agricoles (INERA) et la FAO, les bonnes pratiques agricoles ont été testées à travers les champs écoles des producteurs (CEP) en comparaison avec les pratiques paysannes entre 2006 et 2009. Les résultats agronomiques obtenus montrent une nette amélioration de la productivité des cultures dans les parcelles de bonnes pratiques agricoles par rapport aux pratiques paysannes. Cependant, les changements édaphiques ayant conduit à ces performances ont reçu peu d'attention de la part du monde scientifique. La présente activité a été initiée dans le but de répondre à cette question. On assume que les bonnes pratiques agricoles entraînent des changements des propriétés physiques, chimiques et biologiques des sols et ces changements sont à la base des performances agronomiques observées.

\section{MATERIEL ET METHODES \\ Présentation du site expérimental}

Les travaux ont été conduits à la station de recherche de Farako-ba dans la zone Ouest du Burkina Faso en 2006, 2007 et 2008. Les coordonnées géographiques de la station sont : $04^{\circ} 20^{\prime}$ Longitude Ouest, $11^{\circ} 06^{\prime}$ Latitude Nord et $405 \mathrm{~m}$ d'altitude. Le climat de la zone est de type sud-soudanien (Guinko, 1984) marqué par l'alternance de deux saisons fortement contrastées: une saison pluvieuse qui dure 5 à 6 mois (mai-octobre) et une saison sèche, qui dure 5 à 6 mois (novembre-avril). La pluviométrie est très variable et la moyenne annuelle sur les 30 dernières années est de 950 $\mathrm{mm}$. La pluviométrie au cours des trois années d'expérimentation a été normale comparée à la moyenne sur les 10 dernières années (Tableau 1). 
Les températures varient entre $17{ }^{\circ} \mathrm{C}$ et $37{ }^{\circ} \mathrm{C}$ en saison sèche et de $20{ }^{\circ} \mathrm{C}$ à $32{ }^{\circ} \mathrm{C}$ en saison humide. L'évapotranspiration est très variable et elle est de $8.7 \mathrm{~mm} /$ jour entre janvier et mars et de $3.7 \mathrm{~mm} /$ jour entre juillet et septembre. Les sols du site sont de type ferrugineux tropical. Ce sont des sols sableux, à texture sablo-limoneuse, légèrement acides et pauvres en azote et en phosphore assimilable (Bado, 2002). Ils sont pauvres en argile et en matière organique, ce qui explique leur faible Capacité d'Échange Cationique (CEC). Le Tableau 2 donne les caractéristiques des sols du site expérimental.

\section{Méthode}

L'expérimentation a consisté à comparer les pratiques des producteurs (PP) avec les bonnes pratiques agricoles (BPA). Les bonnes pratiques agricoles ont été définies comme étant «des pratiques qui permettent de satisfaire les besoins actuels, d'améliorer les moyens d'existence, tout en préservant l'environnement de façon durable». De façon spécifique cette notion fait appel a) à une gestion durable de la fertilité des sols, b) une lutte intégrée des ravageurs des cultures c) la maîtrise des itinéraires techniques des cultures (FAO, 2005). Les pratiques paysannes correspondent aux techniques actuelles de gestion des exploitations utilisées par les producteurs dans la zone sud-soudanienne du Burkina Faso.

Le dispositif expérimental utilisé est un split-plot avec les pratiques dans les parcelles principales et les cultures dans les parcelles secondaires. Les traitements ont été regroupés dans 4 blocs de $465 \mathrm{~m}^{2}$ correspondant aux répétitions ; séparés par des allées de $2 \mathrm{~m}$. Les parcelles principales étaient séparées par des allées de $1 \mathrm{~m}$ et les parcelles secondaires de $0,5 \mathrm{~m}$. Le matériel végétal utilisé dans le dispositif était composé du maïs (variété SR21; cycle : 95-110 jours); du coton (variété FK37, cycle 120-140 jours) et du Mucuna (variété deeringiana, cycle: 150 jours). Les pratiques (PP et BPA) ont été utilisées dans un système de rotation culturale maïs-mucuna-coton. Le Tableau 3 donne les spécifications par pratique et par culture. En plus du Mucuna deeringiana, le Stylosanthes hamata et le Cajanus cajan (pois d'angole) ont été utilisés sur des parcelles de $100 \mathrm{~m}^{2}$ sur des parcelles voisines comme plantes couverture en guise d'alternative au Mucuna deeringiana. Ces plantes de couverture ont été choisies à cause de leur adaptation aux conditions locales et leur impact sur la fertilité des sols.

Le maïs et le coton ont été fertilisés selon les doses recommandées pour ces cultures: soit pour le maïs : $200 \mathrm{~kg} \mathrm{NPK} / \mathrm{ha}$ au semis et $150 \mathrm{~kg} / \mathrm{ha}$ d'urée aux $30{ }^{\text {ème }}$ et $45^{\text {ème }}$ jours après semis; pour le coton : $150 \mathrm{~kg}$ $\mathrm{NPK} / \mathrm{ha}$ au semis et $50 \mathrm{~kg} / \mathrm{ha}$ d'urée au $40^{\text {ème }}$ jour après semis. Les plantes de couverture n'ont pas été fertilisées.

Les écartements de semis ont été $80 \mathrm{~cm}$ x $40 \mathrm{~cm}$ pour le cotonnier et le maïs, de $40 \mathrm{~cm}$ x $40 \mathrm{~cm}$ pour les plantes de couverture (Mucuna, Stylosanthes et Cajanus). Pour la protection du cotonnier contre les ravageurs, des applications d'insecticides ont été effectuées tous les 14 jours à partir du $40^{\text {ème }}$ jour après les semis.

La mise en œuvre des activités s'est faite en s'appuyant sur l'approche Champ Ecole des Producteurs selon la FAO. Le Champ Ecole Paysan (CEP) est un cadre restreint d'échanges et de formation participative où un groupe de producteurs ayant un intérêt commun, recherche des solutions, discute et prend des décisions par rapport à des contraintes de production. Le CEP est une école sans «mur» qui réunit les producteurs une fois par semaine pendant une campagne entière de culture. Ils travaillent sous la supervision d'un agent de vulgarisation qui joue plutôt le rôle de facilitateur. Le CEP est un système de formation qui valorise l'expertise paysanne en associant le producteur à toutes les étapes de la formation depuis le diagnostic des problèmes, l'identification et la mise en œuvre des meilleures solutions ainsi que l'évaluation des résultats obtenus. 


\section{Echantillonnage}

Avant la mise en place de l'essai des échantillons de sols ont été régulièrement prélevés chaque année dans les parcelles sur l'horizon 0-20 cm. Les échantillons de sols ont été séchés à température ambiante puis au soleil et dans la hotte, broyés et tamisés à 0,2 $\mathrm{mm}$. Les rendements grains du coton et du maïs ont été évalués par la pose de carré de rendement.

\section{Analyse physico-chimique}

Les échantillons de sols ont été analysés au Laboratoire sols-eaux-plantes de la station de recherche de Farakoba. Le pH eau des échantillons a été déterminé par un $\mathrm{pH}$-mètre (WTW 521) dans une suspension compost-eau de ratio 1:2,5 Le $\mathrm{C}$ total, le $\mathrm{N}$ total, le $\mathrm{P}$ total et assimilable ont été déterminés respectivement selon les méthodes WalkeyBlack (Black, 1965), Kjeldahl et Bray I. Le K total a été déterminé par spectrophotométrie.

\section{Analyse Statistique}

L'ensemble des données collectées a été saisie dans le tableur Excel et l'analyse de variance réalisée avec le logiciel GenStat (GenStat Release 8 for PC/Windows XP, Lawes Agricultural Trust 2005). La séparation des moyennes a été faite en utilisant la plus petite différence significative (LSD) au seuil de probabilité de $5 \%$.

\section{RESULTATS}

Nature de la plante de couverture et propriétés chimiques des sols

Les résultats montrent des propriétés de sols différents en fonction de la nature de la jachère (Tableau 4). Le taux de carbone est significativement plus élevé pour les jachères à Stylosanthes hamata et à Cajanus cajan par rapport aux deux autres jachères. Les mêmes tendances sont observées pour l'azote total. Le rapport $\mathrm{C} / \mathrm{N}$ est comparable pour les jachères à Cajanus cajan, à Mucuna deeringiana et naturelles mais inférieur au rapport obtenu pour la jachère à Stylosanthes hamata. Le phosphore total est identique pour l'ensemble des jachères; par contre le phosphore assimilable est significativement plus élevé pour la jachère naturelle par rapport aux autres jachères. Le plus faible taux de phosphore assimilable est observé dans la jachère à Cajanus cajan. Le Tableau 4 montre des résultats statistiquement identiques pour la variable potassium total $\mathrm{du}$ sol pour l'ensemble des jachères. Les résultats montrent des sols acides avec des $\mathrm{pH}$ inférieurs à 6 pour l'ensemble des jachères. Le $\mathrm{pH}$ eau est statistiquement plus bas dans la jachère naturelle par rapport aux jachères améliorées avec les plantes de couverture.

\section{Propriétés des sols en fonction des pratiques culturales et de la durée de mise en culture}

Les résultats sont statistiquement différents selon les pratiques et la durée de mise en culture. Les taux de matière organique, d' $\mathrm{N}$ total et de $\mathrm{P}$ assimilable sont plus élevés pour les bonnes pratiques agricoles par rapport aux pratiques paysannes (Tableaux 5 et 6). La pratique n'a cependant pas affecté le taux de $\mathrm{K}$ total et le $\mathrm{pH}$ du sol (Tableaux 5 et 6). Les résultats sont comparables pour les campagnes agricoles 2007 et 2008 mais nettement inférieurs aux résultats obtenus en 2009. Le pH n'a pas été affecté avec la durée de mise en culture.

\section{Evolution des propriétés chimiques des sols en fonction de la culture précédente}

Les résultats montrent des différences significatives entre les pratiques et le précédent cultural pour les caractéristiques des sols (Tableaux 7 et 8). Cependant, aucune corrélation positive n'a été observée entre les deux facteurs. Le taux de matière organique est plus élevé pour les précédents mucuna, coton et maïs par rapport à la jachère naturelle. La teneur en $\mathrm{K}$ total et en $\mathrm{P}$ total est statistiquement plus élevée pour le maïs et le coton par rapport aux deux autres précédentes. 
Effet des pratiques sur les rendements coton et maïs grain

Les rendements coton et maïs grains varient énormément avec la culture et la pratique (Tableaux 9 et 10). Pour les deux cultures, les BPA ont permis de récolter deux à trois fois plus de grains par rapport aux pratiques paysannes selon les années. Dans le cas spécifique du coton, on note une grande variabilité des rendements d'une année à l'autre. Les plus faibles rendements coton grain sont enregistrés en 2009 pour les deux pratiques. Les rendements maïs grains présentent des tendances différentes. Les meilleurs rendements maïs grain pour le traitement: pratiques paysannes sont enregistrés en 2009. Les plus faibles rendements maïs grain sont observés en 2008 pour les pratiques paysannes et les bonnes pratiques agricoles. Les rendements obtenus dans les bonnes pratiques agricoles sont nettement supérieurs à la moyenne régionale.

Tableau 1: Pluviométrie de Farakoba de 2006 à 2008 et moyenne sur les 10 dernières années avant l'étude.

\begin{tabular}{lcccc}
\hline \multicolumn{5}{c}{ Pluviométrie (mm) } \\
\hline Monenne (1993-2004) & $\mathbf{2 0 0 6}$ & $\mathbf{2 0 0 7}$ & $\mathbf{2 0 0 8}$ \\
Février & 0,1 & 0 & 0 & 0 \\
Mars & 0,8 & 0 & 0 & 0 \\
Avril & 15,2 & 0 & 0,8 & 4,4 \\
Mai & 68,5 & 71,7 & 79,4 & 0 \\
Juin & 113,0 & 152 & 38,6 & 74,2 \\
Juillet & 126,2 & 160,9 & 128 & 134,7 \\
Août & 217,2 & 177,5 & 248,9 & 304,4 \\
Septembre & 276,4 & 344 & 367,1 & 312,3 \\
Octobre & 184,3 & 268,2 & 165,7 & 250,4 \\
Novembre & 65,4 & 97,7 & 59,1 & 53,8 \\
Décembre & 9,9 & 0 & 26 & 0 \\
Total & 0,7 & 0 & 0 & 0 \\
\hline
\end{tabular}

Tableau 2 : Caractéristiques physico-chimiques des sols de Farakô-Ba. Echantillons composites $(\mathrm{n}=10)$, prélèvement sur l'horizon $0-20 \mathrm{~cm}$.

\begin{tabular}{lc}
\hline Caractéristiques & Valeur \\
\hline Granulométrie & \\
\hline Argile (\%) & 8 \\
Limon (\%) & 12 \\
Sable (\%) & 80 \\
Acidité & \\
pH eau & 6.5 \\
pH KCl & 5.6 \\
Composition chimique & \\
Matière organique totale $(\%)$ & 0.61 \\
C/N & 12 \\
N total (\%) & 0,03 \\
P Bray I (mg Kg & \\
& 2,6
\end{tabular}


P total $\left(\mathrm{mg} \mathrm{Kg}^{-1}\right)$

$\mathrm{K}$ total $\left(\mathrm{mg} \mathrm{Kg}^{-1}\right)$

Bases échangeables et CEC

$\mathrm{Ca}$ échangeable $\left(\mathrm{C} \mathrm{mol}^{+} \mathrm{Kg}^{-1} \mathrm{sol}\right) \quad 1.08$

$\mathrm{K}$ échangeable $\left(\mathrm{C} \mathrm{mol}^{+} \mathrm{Kg}^{-1} \mathrm{sol}\right) \quad 0.02$

$\mathrm{Mg}$ échangeable $\left(\mathrm{C} \mathrm{mol}^{+} \mathrm{Kg}^{-1} \mathrm{sol}\right) \quad 0.46$

$\mathrm{CEC}\left(\mathrm{C} \mathrm{mol}^{+} \mathrm{Kg}^{-1} \mathrm{sol}\right) \quad 1.82$

Acidité d'échange $\left(\mathrm{C} \mathrm{mol}^{+} \mathrm{Kg}^{-1} \mathrm{sol}\right) \quad 0.08$

Saturation en bases (\%) 96

Source : Traoré et al., 2007

Tableau 3 : Contenu des pratiques et selon les cultures.

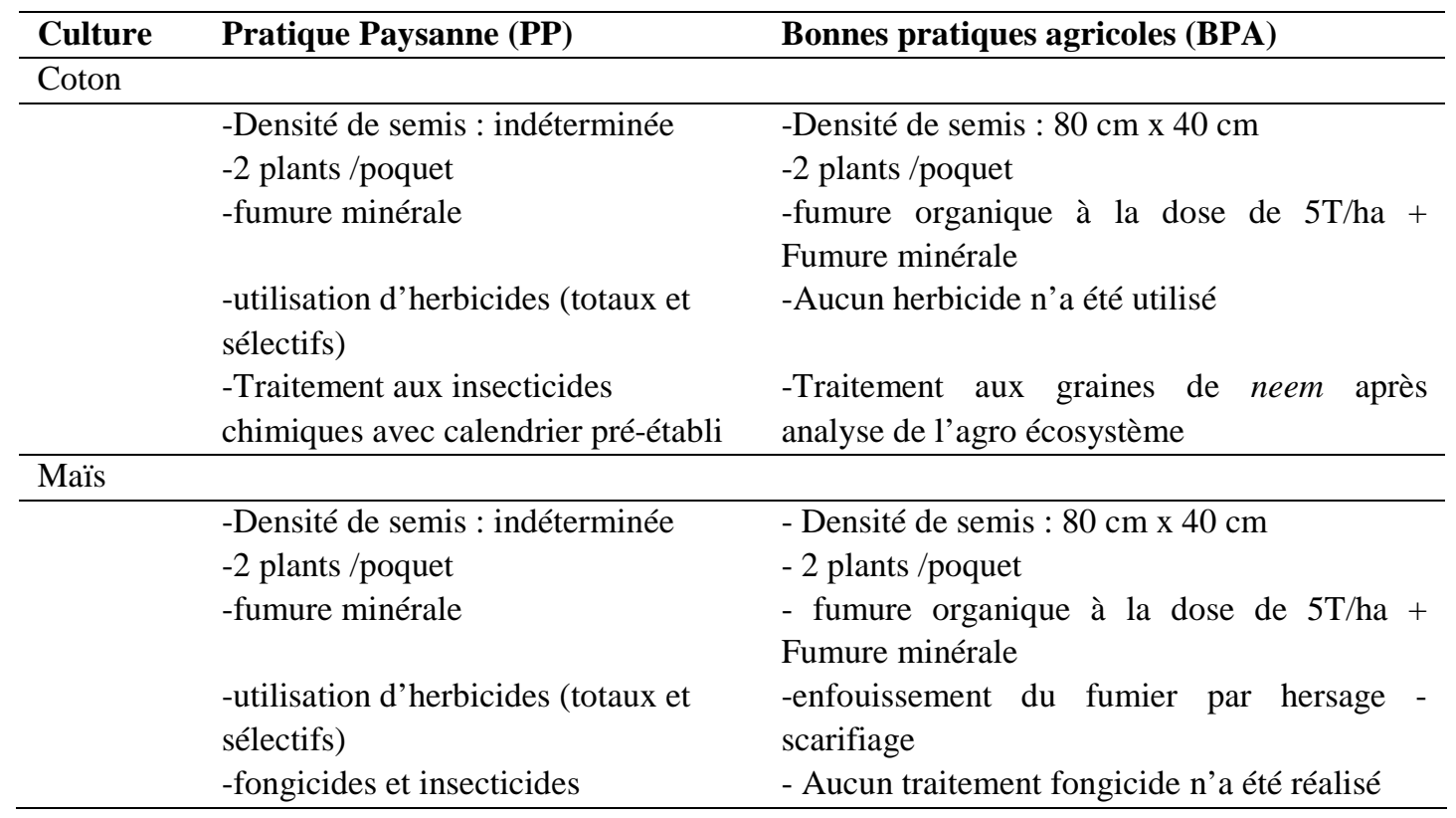

Tableau 4 : Nature de la jachère et propriétés chimiques des sols. Echantillons composite $(n=5$ pour toutes les jachères) prélevés sur l'horizon 0-20 cm. Farakoba 2007.

\begin{tabular}{|c|c|c|c|c|c|}
\hline & \multicolumn{5}{|c|}{ Nature de la jachère } \\
\hline & $\begin{array}{c}\text { Cajanus } \\
\text { cajan }\end{array}$ & $\begin{array}{c}\text { Jachère } \\
\text { naturelle }\end{array}$ & $\begin{array}{c}\text { Mucuna } \\
\text { deeringiana }\end{array}$ & $\begin{array}{c}\text { Stylosanthes } \\
\text { hamata }\end{array}$ & Probabilité \\
\hline $\mathrm{C}(\%)$ & 0,41 & 0,33 & 0,32 & 0,46 & 0,013 \\
\hline $\mathrm{N}(\%)$ & 0,035 & 0,031 & 0,031 & 0,04 & 0,045 \\
\hline $\mathrm{C} / \mathrm{N}$ & 10,6 & 10,8 & 9,93 & 12,0 & 0,005 \\
\hline $\mathrm{P}$ total $\left(\mathrm{mg} \mathrm{kg}^{-1}\right)$ & 64,4 & 76,6 & 77,5 & 86,9 & 0,70 \\
\hline $\mathrm{P}$ assimilable $\left(\mathrm{mg} \mathrm{kg}^{-1}\right)$ & 1,53 & 4,48 & 2,39 & 2,41 & $<0,001$ \\
\hline K_total $\left(\mathrm{mg} \mathrm{kg}^{-1}\right)$ & 316,4 & 447,7 & 491,4 & 440,1 & 0,131 \\
\hline $\mathrm{pH}_{\text {eau }}$ & 5,89 & 5,15 & 5,75 & 5,73 & 0,002 \\
\hline $\mathrm{pH}_{\mathrm{KCl}}$ & 4,69 & 4,26 & 4,56 & 4,66 & 0,159 \\
\hline
\end{tabular}


Tableau 5 : Caractéristiques chimiques du sol en fonction des pratiques et des années de mise en culture. Echantillons prélevés sur l'horizon 0-20 cm ( $\mathrm{n}=5$ échantillons/pratique/an).

\begin{tabular}{|c|c|c|c|c|c|c|}
\hline & \multicolumn{6}{|c|}{ Pratiques culturales } \\
\hline & \multicolumn{3}{|c|}{$\mathbf{P P}^{1}$} & \multicolumn{3}{|c|}{$\overline{\mathbf{B P A}^{2}}$} \\
\hline & 2007 & 2008 & 2009 & 2007 & 2008 & 2009 \\
\hline Matière Organique (\%) & 0,57 & 0,56 & 1,01 & 0,67 & 0,68 & 1,18 \\
\hline $\mathrm{N}$ total $(\%)$ & 0,032 & 0,028 & 0,044 & 0,039 & 0,036 & 0,054 \\
\hline $\mathrm{C} / \mathrm{N}$ & 10,3 & 11,4 & 13,4 & 10,1 & 11,1 & 13,0 \\
\hline $\mathrm{K}$ total $\left(\mathrm{mg} \mathrm{kg}^{-1}\right)$ & 417,5 & 459,6 & 563,7 & 455,6 & 437,9 & 529 \\
\hline $\mathrm{P}$ assimilable $\left(\mathrm{mg} \mathrm{kg}^{-1}\right)$ & 3,1 & 2,6 & 3,7 & 10,71 & 7,48 & 8,07 \\
\hline $\mathrm{P}$ total $\left(\mathrm{mg} \mathrm{kg}^{-1}\right)$ & 81,6 & 78,7 & 116,3 & 92,6 & 91,4 & 127,7 \\
\hline $\mathrm{pH}$ eau & 5,68 & 5,67 & 5,59 & 5,58 & 5,75 & 5,59 \\
\hline $\mathrm{pH}_{\mathrm{KCl}}$ & 4,53 & 4,34 & 4,19 & 4,47 & 4,47 & 4,23 \\
\hline
\end{tabular}

Tableau 6: Résultats de l'analyse de variance sur le changement des caractéristiques chimiques des sols en fonction des pratiques et des années de mise en culture.

\begin{tabular}{lccc}
\hline Variables & \multicolumn{3}{c}{ Probabilité } \\
\cline { 2 - 4 } & Année & pratique & Pratique x année \\
\hline Matière Organique (\%) & $<.001(0,087)$ & $<.001(0,071)$ & $\mathrm{NS}$ \\
$\mathrm{N}$ total $(\%)$ & $<.001(0,004)$ & $<.001(0,003)$ & $\mathrm{NS}$ \\
$\mathrm{C} / \mathrm{N}$ & $<.001(0,88)$ & $\mathrm{NS}$ & $\mathrm{NS}$ \\
$\mathrm{K}$ total $\left(\mathrm{mg} \mathrm{kg}^{-1}\right)$ & $<.001(42,8)$ & $\mathrm{NS}$ & $\mathrm{NS}$ \\
$\mathrm{P}$ assimilable $\left(\mathrm{mg} \mathrm{kg}^{-1}\right)$ & $\mathrm{NS}$ & $<.001(1,83)$ & $\mathrm{NS}$ \\
$\mathrm{P}$ total $\left(\mathrm{mg} \mathrm{kg}^{-1}\right)$ & $<.001(8,98)$ & $0,002(7,34)$ & $\mathrm{NS}$ \\
$\mathrm{pH}$ & $\mathrm{NS}$ & $\mathrm{NS}$ & $\mathrm{NS}$ \\
$\mathrm{pH}$ & $\mathrm{KCl}$ & $\mathrm{NS}$ & $\mathrm{NS}$ \\
\hline *ntre parenthèse $=$ LSD & & &
\end{tabular}


Tableau 7 : Propriétés chimiques des sols en fonction de la culture précédente. Echantillons composites ( $\mathrm{n}=5$ par pratique et par type de jachère), prélèvement sur l'horizon $0-20 \mathrm{~cm}$. Farakoba 2007, 2008, 2009.

\begin{tabular}{|c|c|c|c|c|c|c|c|c|}
\hline & \multicolumn{8}{|c|}{ Pratiques culturales } \\
\hline & \multicolumn{4}{|c|}{ PP } & \multicolumn{4}{|c|}{ BPA } \\
\hline & Jachère & Coton & Mucuna & Maïs & Jachère & Coton & Mucuna & Maïs \\
\hline Matière Organique (\%) & 0,57 & 0,77 & 0,78 & 0,79 & 0,67 & 0,96 & 0,98 & 0,83 \\
\hline $\mathrm{N}$ total $(\%)$ & 0,032 & 0,037 & 0,033 & 0,039 & 0,039 & 0,042 & 0,048 & 0,044 \\
\hline $\mathrm{C} / \mathrm{N}$ & 10,3 & 11,9 & 13,6 & 11,7 & 10,1 & 13,2 & 11,9 & 11 \\
\hline $\mathrm{K}$ total $\left(\mathrm{mg} \mathrm{kg}^{-1}\right)$ & 418 & 519 & 482 & 534 & 456 & 474 & 463 & 514 \\
\hline $\mathrm{P}$ assimilable $\left(\mathrm{mg} \mathrm{kg}^{-1}\right)$ & 3,1 & 3,14 & 4,05 & 2,34 & 10,7 & 8,77 & 8,5 & 6,06 \\
\hline $\mathrm{P}$ total $\left(\mathrm{mg} \mathrm{kg}^{-1}\right)$ & 81,6 & 98 & 97,6 & 97 & 92,6 & 113,8 & 104,4 & 110,5 \\
\hline $\mathrm{pH}_{\text {eau }}$ & 5,68 & 5,61 & 5,63 & 5,66 & 5,58 & 5,81 & 5,68 & 5,51 \\
\hline $\mathrm{pH}_{\mathrm{KCl}}$ & 4,53 & 4,23 & 4,25 & 4,3 & 4,47 & 4,52 & 4,37 & 4,16 \\
\hline
\end{tabular}

Tableau 8 : Résultats de l'analyse de variance sur les propriétés chimiques en fonction de la culture précédente.

\begin{tabular}{lccc}
\hline Variable & \multicolumn{3}{c}{ Probabilité } \\
\hline & Pratique & Précédent & Pratique x Précédent \\
\hline Matière Organique (\%) & $0,034(0,12)$ & $0,005(0,16)$ & $\mathrm{NS}$ \\
$\mathrm{N}$ total $(\%)$ & $0,003(0,005)$ & $\mathrm{NS}$ & $\mathrm{NS}$ \\
$\mathrm{C} / \mathrm{N}$ & $\mathrm{NS}$ & $<.001(1,03)$ & $\mathrm{NS}$ \\
$\mathrm{K}$ total $\left(\mathrm{mg} \mathrm{kg}^{-1}\right)$ & $\mathrm{NS}$ & $0,016(54,8)$ & $\mathrm{NS}$ \\
$\mathrm{P}$ assimilable $\left(\mathrm{mg} \mathrm{kg}^{-1}\right)$ & $<.001(1,82)$ & $\mathrm{NS}$ & $\mathrm{NS}$ \\
$\mathrm{P}$ total $\left(\mathrm{mg} \mathrm{kg}^{-1}\right)$ & $0,033(10,7)$ & $0,041(14,6)$ & $\mathrm{NS}$ \\
$\mathrm{pH}$ & $\mathrm{NS}$ & $\mathrm{NS}$ & $\mathrm{NS}$ \\
$\mathrm{pH}$ & $\mathrm{KCl}$ & $\mathrm{NS}$ & $\mathrm{NS}$ \\
\hline \multicolumn{2}{c}{$1:$ Entre parenthèse $=\mathrm{LSD} ;$} & $2: \mathrm{NS}=$ non significatif &
\end{tabular}

Tableau 9 : Rendement coton graine et maïs grain en fonction des pratiques et de la durée de mise en culture.

\begin{tabular}{lcccc}
\hline & \multicolumn{3}{c}{ Rendement des cultures $\left(\mathrm{kg} \mathrm{ha}^{-1}\right)$} \\
\hline \multicolumn{3}{c}{ Coton } & \multicolumn{2}{c}{ Maïs } \\
\hline Année & PP & BPA & PP & BPA \\
\hline 2007 & 947 & 2086 & 1445 & 4250 \\
2008 & 1034 & 1960 & 1392 & 3616 \\
2009 & 530 & 1640 & 1705 & 4097 \\
\hline
\end{tabular}


Tableau 10 : Résultats de l'analyse de variance sur les rendements des cultures.

\begin{tabular}{lll}
\hline Facteur & Pr & LSD \\
\hline Année & NS & 345 \\
\hline Culture & $<0,001$ & 282 \\
Pratique & $<0,001$ & 282 \\
Année x culture & NS & 488 \\
Année x pratique & NS & 488 \\
culture x pratique & $<, 001$ & 399 \\
Année x culture x pratique & NS & 690 \\
\hline
\end{tabular}

\section{DISCUSSION}

\section{La jachère annuelle améliorée et propriétés des sols}

Les jachères à stylosanthes et à pois d'angole ont permis d'accumuler plus de carbone dans les sols, cela est attribuable aux caractéristiques physiologiques de ces plantes améliorantes et à leur capacité à produire de la biomasse. En effet, le stylosanthes et le pois d'angole sont des légumineuses très lignifiées et produisent plus de biomasse que la jachère naturelle (Becker et Johnson, 1998). La jachère à Stylosanthes à permis d'augmenter significativement le taux d'azote du sol. Ces résultats sont similaires à ceux rapportés par Bado (2002). Cet auteur indique qu'une année de légumineuse était nettement plus profitable qu'une jachère naturelle annuelle sur le plan de la restauration de la fertilité des sols. Par contre aucune des jachères n'a affecté le $\mathrm{pH}$ du sol. Ce facteur est peu affecté par une jachère annuelle et seule une jachère longue durée permet de restaurer l'ensemble des propriétés des sols (Somé et al., 2007). Le niveau du phosphore assimilable dans les jachères est nettement en dessous du seuil de déficience établi à $5 \mathrm{mg} / \mathrm{kg}$ de sol (Compaore et al., 2001). Ce faible taux de P assimilable est en accord avec l'acidité élevée des sols (Lompo, 1995; Sedogo, 1993). Ce qui nécessitera des sources extérieures de phosphore pour atteindre des niveaux adéquats de phosphore assimilable dans le sol (Sanchez, 2002). De ces résultats il ressort que la valorisation des jachères améliorées nécessitera l'utilisation d'une source extérieure de phosphore en vue de productions potentielles. Le stylosanthes et le pois d'angole pourraient être utilisés dans les rotations au vue de leur performance, cependant le rythme de la couverture de ces plantes est beaucoup plus lente que dans le cas du muсuna (données non présentées ici). Une vision pour le long terme devrait intégrer une plante de couverture lignifiée et une plante à décomposition facile.

\section{Les pratiques (PP et BPA) et la gestion de la fertilité des sols}

Le taux de matière organique est plus élevé dans les parcelles de bonnes pratiques agricoles probablement à cause de l'apport de $5 \mathrm{t}$ de fumure organique/ha dans ces parcelles. Cependant, l'utilisation de la fumure organique n'a pas permis de relever le $\mathrm{pH}$ du sol de façon significative comme rapporté par Joann et al. (2000). La différence pourrait être liée aux doses de fumures organiques utilisées dans les deux expérimentations. En effet, ces auteurs ont utilisé des doses de 40 t/ha soit près de 10 fois la dose utilisée dans notre expérimentation.

L'utilisation de la fumure organique a par contre permis une meilleure disponibilité du $\mathrm{P}$ dans le sol probablement grâce à un meilleur fonctionnement du complexe argilohumique. 
En plus du taux de matière organique et de $\mathrm{P}$ assimilable, les bonnes pratiques ont significativement affecté la teneur en azote des sols. L'amélioration significative du taux de $\mathrm{P}$ assimilable et d' $\mathrm{N}$ est un résultat très important pour la production agricole au niveau du sahel où l'N et le $\mathrm{P}$ constituent les facteurs les plus limitant pour la production agricole (Sedogo, 1993; Lompo, 1995 ; Bationo et al., 1998 ; Traoré et Stroosnijder, 2005 ; Traoré et al., 2007). De ce fait la productivité des parcelles des bonnes pratiques agricoles sera nettement améliorée.

Les résultats obtenus montrent que la nature du précédent cultural affecte le taux de matière organique. Le plus faible taux de matière organique est obtenu dans la jachère naturelle annuelle. Ces résultats sont en corrélation avec ceux indiqués précédemment. Cette différence est liée à la faible quantité de biomasse produite dans les jachères naturelles annuelles (Becker et Johnson, 1998). La teneur en azote des sols est restée la même pour l'ensemble des précédents. Ces résultats sont en désaccord avec ceux de Bado (2002) qui rapportent qu'une jachère à légumineuse permettait d'accumuler plus d'N dans le système que les autres précédents. Le gain d'N dans les parcelles à mucuna a été probablement masqué par l'utilisation des engrais azotés dans les autres précédents. Les résultats montrent que ni le précédent cultural ni la pratique ne permettent de changer significativement le $\mathrm{pH}$ du sol. Ces résultats laissent transparaître l'obligation d'utiliser des amendements (chaux vive ou éteinte, dolomie) pour baisser significativement le $\mathrm{pH}$ du sol. A défaut de ces amendements, de fortes doses de matière organique auraient été indispensables (Joann et al., 2000) ce qui n'est pas envisageable pour de petits producteurs et dans un contexte de pauvreté.

\section{Pratiques (PP, BPA) et rendements des cultures}

Les rendements coton et maïs grain ont été nettement améliorés par les bonnes pratiques agricoles par rapport aux pratiques paysannes. Les gains de rendements observés pour ces parcelles BPA sont probablement liés à l'amélioration des conditions pédologiques; spécialement l'amélioration de la teneur en $\mathrm{P}$ assimilable et en $\mathrm{N}$ de ces parcelles BPA (Lavigne-Delville, 1996; Bationo et al., 1998). Ces résultats obtenus sont en parfaite concordance avec ceux obtenus pour les sols. La durabilité des systèmes de production dans cette zone dépendra fortement de la capacité à maintenir le taux de phosphore assimilable au dessus du seuil de déficience de $5 \mathrm{mg} \mathrm{kg}^{-1}$ de sol comme l'on rapporté Compaoré et al. (2001).

Les rendements ont connu une variation saisonnière importante. Ces résultats sont imputables aux conditions climatiques qui sont variables d'une année à une autre. Des résultats similaires sont rapportés par plusieurs auteurs pour la même zone (Guillobez et Zougmoré, 1991; Fournier et al., 2000 ; Somé et al., 2000). Les BPA apparaissent alors comme un paquet technologique capable de soutenir la production à long terme.

\section{Conclusions}

De nos travaux il ressort que les jachères à stylosanthes et à pois d'angole permettent d'améliorer la fertilité des sols par rapport à la jachère naturelle. Ces jachères améliorées affectent significativement le taux de matière organique et de phosphore assimilable du sol à des niveaux plus importants que pour le mucuna.

Les résultats ont montré également que les bonnes pratiques agricoles permettent d'améliorer les teneurs des sols en matière organique, en azote et en phosphore assimilable par rapport à la pratique paysanne. Ces résultats sont très importants pour la zone tropicale où la productivité des sols est fortement limitée par la disponibilité en ces éléments nutritifs. Cependant, les BPA n'ont pas affecté significativement le $\mathrm{pH}$ du sol ainsi que la teneur en potassium du sol. Pour la durabilité des systèmes de production, un accent doit être mis sur les sources de 
potassium (utilisation de cendre dans la matière organique apportée). Il sera également nécessaire de prévoir l'utilisation d'un amendement en vue de réduire le $\mathrm{pH}$ du sol (chaux ou dolomie). Les BPA ont permis d'obtenir des rendements coton graine et maïs grain significativement plus importants par rapport à la pratique paysanne. Les grains de rendement varient d'une année à l'autre suite à des conditions climatiques variables. La mise en place de techniques de conservation des eaux et des sols permettrait de stabiliser des parcelles soumises aux effets du climat (érosion hydrique essentiellement).

\section{REFERENCES}

Bado BV.2002. Rôle des légumineuses sur la fertilité des sols ferrugineux tropicaux des zones guinéennes et soudaniennes du Burkina Faso. Thèse de Ph.D Département des sols et Environnement, Université Laval, 145 p.

Bationo A, Lompo F, Koala S. 1998. Research on nutrient flows and balances in West Africa: state of the art. Agriculture, Ecosystems and Environment, 71: 19-35.

Becker M, Johnson D. 1998. Les légumineuses comme jachères de saison sèche dans des systèmes de riz pluvial en Afrique de l'Ouest. Biology and Fertility of Soils, 27: 358-367

Black CA. 1965. Methods of soil analysis; part 2. Chemical and microbiological properties. Soil Science Society of America; 1367-1375.

Compaoré E, Fardeau JC, Morel JL, Sedogo, MP. 2001. Le phosphore biodisponible des sols: une des clés de l'agriculture durable en Afrique de l'Ouest. Cahiers d'Etudes et de Recherche Francophone/Agriculture, 10(2): 81-85.

Fournier A, Serpantié G, Delhoum JP, Gattelier R. 2000. Rôle des jachères sur les écoulements de surface et l'érosion en zone soudanienne du Burkina Faso. Application à l'aménagement des versants. In La Jachère en Afrique
Tropicale, Floret et Pontanier (eds). John Libbey Eurotext ; 179-188.

Guillobez S, Zougmoré R. 1991. Etude du ruissellement et de ses principaux paramètres à la parcelle (Saria, Burkina Faso). In Bilan Hydrique Agricole et Sécheresse en Afrique Tropicale. Ed. John Libbey Eurotext : Paris ; 319-329.

Guinko S. 1984. Végétation de la Haute Volta. Thèse de doctorat ès sciences naturelles, Université Bordeaux III, Tome 1. France. 318 p.

INERA.1994. Les systèmes de production dans la zone Ouest du Burkina Faso: potentialités, contraintes, bilan et perspectives de recherche. INERA, Ouagadougou, Burkina Faso, 48p.

INERA. 2003. Recherche sur des technologies de lutte contre la désertification au sahel et étude de leur impact agro écologique. INERA, Département GRN/SP Burkina Faso, 91p.

Joann K, Whalen, Chi Chang, George WC, Janna PC. 2000. Cattle Manure Amendments Can Increase the $\mathrm{pH}$ of Acid Soils. Soil Sci. Soc. Am. J., 64: 962966.

Lavigne-Delville. 1996. Gérer la fertilité des terres dans les pays du Sahel. Diagnostic et Conseil aux paysans. Collection « Le Point sur », 397 p.

Lompo F. 1995. Etude de cas au Burkina Faso de l'initiative phosphates naturels. INERA/Burkina Faso, Rapport de travail, $36 \mathrm{p}$.

Sanchez PA 2002. Ecology - Soil fertility and hunger in Africa. Science, 295(5562): 2019.

Sédogo PM. 1981. Contribution à la valorisation des résidus culturaux en sol ferrugineux et sous climat tropical semiaride (matière organique $\mathrm{du}$ sol et nutrition azotée des cultures). Thèse de docteur Ingénieur sciences agronomiques, Institut National Polytechnique de lorraine-Nancy, 198 p.

Sédogo PM. 1993. Evolution des sols ferrugineux lessivés sous culture : 
incidence des modes de gestion sur la fertilité. Thèse de doctorat, Univ. Cocody. C.I., 285 p.

Some L, Kambou F, Traore S, Ouedraogo B. 2000. Water and soil conservation techniques in northern Burkina Faso. Science et changements planétaires / Sécheresse. Water and Water Management, 11: 267-274.

Somé NA, Traoré K, Traoré O, Tassembédo M. 2007. Potentiel des jachères artificielles à Andropogon sp. dans l'amélioration des propriétés chimiques et biologiques des sols en zone soudanienne (Burkina Faso). Biotechnologie, Agronomie, Société, Environnement (BASE), 11(3): 245-252.

Thiombiano L. 2000 : Etude de l'importance des facteurs édaphiques et pédopaysagiques dans le développement de la désertification en zone sahélienne du Burkina Faso. Thèse d'Etat, Université de Ouagadougou, Burkina Faso, volume 1, 209 p.

Traoré K, Stroosnijder L. 2005. Sorghum quality, organic matter amendments and health; farmers' perception in Burkina Faso, West Africa. Ecology of Food and Nutrition, 44: 225-245.
Traoré O, Traoré K, Bado BV, Lompo DJP. 2007. Crop rotation and soil amendments: impacts on cotton and maize production in a cotton based system in western Burkina Faso. Int. J. Biol. Chem. Sci., 1(2): 143-150.

Vlaar. 1992. Les techniques de conservation des eaux et des sols dans les pays du Sahel. CIEH, Burkina Faso, Université Agronomique de Wageningen, $99 \mathrm{p}$.

Zombré NP, Mando A, Ilboudo JB, 1999. Impact des conservations des eaux et des sols sur la restauration des jachères très dégradées au Burkina Faso. In La Jachère en Afrique de l'Ouest. Rôles, Aménagements et Alternatives (vol. 1). Floret et Pontanier (éd., 2000); pp. 771777.

Zougmoré R, Zida Z, Kambou FN. 1999: Réhabilitation des sols dégradés: rôles des amendements dans le succès des techniques de demi-lune et de zaï au Sahel. Bulletin Réseau Erosion 19: L'influence de l'homme sur l'érosion, volume 1 à l'échelle du versant; $p$ 536549 . 